\title{
Evaluación del consumo de antimicrobianos en una Unidad de Neonatología: un trabajo en equipo para promover el uso racional de antimicrobianos
}

\author{
Elisa Jiménez, Nicolás Valls, Patricio Astudillo, Cristián Valls, Gabriel Cavada, Alejandra Sandoval, \\ Angélica Alegría, Gabriela Ortega, Daniela Núñez y Patricia Mena
}

Complejo Asistencial Dr. Sótero del Río, Santiago, Chile. Servicio Neonatología (EJA, GO, DN, PM).

Servicio de Pediatría (AS). Facultad de Medicina, Universidad de Chile, Santiago, Chile. Instituto de Ciencias Biomédicas, Laboratorio de estrés oxidativo (NV).

Departamento de Salud Pública (GC). Médico-Cirujano Universidad Católica (PA). Pontificia Universidad Católica de Chile, Santiago, Chile Facultad de Ciencias, Departamento Biología Celular y Molecular (CV).

Clínica Dávila, Santiago, Chile. Servicio Neonatología (AA)

Lugar de realización: Servicio de Recién Nacidos Complejo Asistencial Dr. Sótero del Río.

Los autores declaran no tener conflictos de interés. No se recibió financiamiento alguno para la realización del estudio.

Recibido: 4 de mayo de 2017 Aceptado: 14 de noviembre de 2017

Correspondencia a: Elisa Jiménez Armaroli soteroneo@gmail.com

\section{Evaluation of antimicrobial consumption in a Neonatology Unit: a team work to promote the rational use of antibiotics}

Background: Antibiotics (ATB) are drugs widely used in hospitalized newborns. The indiscriminate use of ATBs promote the rise of resistant bacteria to the most commonly indicated antimicrobials. In addition, ATB prescription presents associations to morbidity, such as bronchopulmonary dysplasia, necrotizing enterocolitis, late sepsis and even death. All of the above leads to an increase in health care costs. Aim: To record and to evaluate trends of antibiotic use over time in hospitalized NB in the Neonatology Unit at Dr. Sótero del Río Hospital, in order to objectify the changes in the usual practice of the ATM indication. A secondary objective was to assess its impact on antimicrobial resistance. Methods: Cohort, observational, prospective unicenter study which included all hospitalized patients between January 2011 and December 2014. Birth weight, hospitalization days, ATB indication and days of ATB use were recorded for each patient. The use of ATB was quantified by means of different rates; days of indication of one or more ATBs for global consumption (RUA), total sum of days of use (TSUA) and for the most frequently used ATBs. Each calculated rate for 100 days hospitalized. In addition, the antimicrobial susceptibility of the most frequently isolated bacteria in our service: coagulase-negative Staphylococcus (SCN) and Gram-negative bacilli (BGN) were recorded continuously. Results: The $34.7 \%$ of the hospitalized patients received some type of antimicrobial agent. ATBs were $32.3 \%$ of medicines used. The most widely used was ampicillin (with $20.2 \%$ of the total) and cefadroxyl (with $11.6 \%$ ). The RUA did not change during the study time, but STUA decreased by $10.7 \%$ between 2011 and 2014 with $p<0.05$. When subgroup analyzes were divided by weight ranges, in the $<750 \mathrm{~g}$ group, the use of vancomycin decreased in use by $9.9 \%$ and an increase of $18.8 \%$ for metronidazole was observed. On the other hand, there was an increase in the use of the piperacillin-tazobactam regimen in the range $>1,500 \mathrm{~g}$. When evaluating antimicrobial susceptibility, there was a decrease in susceptibility for oxacillin in SCN between 2011 and 2014 from $27 \%$ to $10.3 \%$ respectively. In addition, for Gram negative there was a decrease from $76.9 \%$ to $40.5 \%$ in susceptibility to third generation cephalosporins, mainly due to Klebsiella pneumoniae, which became the predominantly isolated BGN with an increase of $6.7 \%$ to $50 \%$ between 2011 and 2014 , respectively. For $K$. pneumoniae the loss of susceptibility to third generation cephalosporins decreased from $77 \%$ to $22 \%$. Finally, amikacin showed an activity over $85 \%$ in all BGNs between 2011 and 2014. Conclusions: It is advisable to plan and to maintain a continuous record of ATB consumption, as well as therapy and prophylaxis, being categorized by ATB type and range of newborn weight. It is of considerable importance to analyze and to evaluate the susceptibility of microorganisms. It is essential that an interdisciplinary team prepare this recording, and to continuously provide feedback to professionals who maintain the functioning of neonatal care units.

Key words: Antimicrobial stewardship, antibiotic, infants, newborn, antibiotic use rates, antibiotic susceptibility.

Palabras clave: Programa de optimización de antimicrobianos, antimicrobianos, recién nacidos, tasa de uso de antimicrobianos, susceptibilidad a antimicrobianos.

\section{Introducción}

L os antimicrobianos (ATMs) son uno de los medicamentos más utilizados en los pacientes de unidades de internación en intensivo neonatal ${ }^{1}$. Según el Centro de Control y Prevención de Enfermedades Infecciosas (CDC) de Atlanta, Georgia, los ATMs son innecesarios o mal utilizados hasta en $50 \%$ de las veces ${ }^{2}$.
En recién nacidos $(\mathrm{RN})$ el diagnóstico por sospecha de infección es difícil, lo que favorece utilizar terapias de ATMs en forma empírica. Las infecciones en esta etapa de la vida tienen una expresión clínica inespecífica, con el agravante de que existen otras enfermedades de presentación muy similar, que pueden ser concomitantes o sobre agregadas ${ }^{3,4}$. Esto incluye a cuadros de etiología viral, los que también se traducen en una presentación 
clínica y laboratorio inespecíficos, e incluso, en un bajo porcentaje, pueden sobreponerse a cuadros de causa bacteriana ${ }^{5-7}$. Adicionalmente, los exámenes de laboratorio para infección son de baja sensibilidad y de bajo poder predictor positivo ${ }^{8}$. En la práctica clínica se aplican esquemas de tratamiento ATM en forma empírica con el fin de cubrir adecuadamente a los microorganismos causales más frecuentes. La elección empírica del tipo de ATM se basa en la epidemiología local y en lo recomendado por la literatura médica; sin embargo, actualmente no existe consenso de la extensión de la duración de la terapia antimicrobiana y los días a emplearlos varían según los distintos centros ${ }^{9}$.

Lo anteriormente expuesto genera un uso indiscriminado de ATMs trayendo consecuencias negativas en forma individual y para el ambiente microbiológico de las unidades de cuidados intensivos neonatales.

En el paciente prematuro extremo, el uso perinatal de ATMs favorece una relativa abundancia de bacterias gramnegativas ${ }^{10,11}$ lo que se asocia a un aumento del riesgo de enterocolitis necrosante, sepsis tardía o incluso muerte ${ }^{11-14}$. De hecho, el dar ATMs a prematuros se ha asociado con aumento de ecogenicidad periventricular, retinopatía del prematuro y de mortalidad ${ }^{12}$. Otro ejemplo del efecto individual asociado al uso de ATM, son las infecciones por hongos secundarias al uso de cefalosporinas de tercera generación, lo que ha llevado a limitar su uso a meningitis $\mathrm{y}$, en forma excepcional, en otras situaciones. El riesgo aumentado de adquirir infecciones por hongos también se ha descrito al utilizar otros ATMs como los carbapenémicos, piperacilina, ticarcilina y vancomicina ${ }^{15-17}$.

El uso de ATMs en el ambiente microbiológico hospitalario se puede traducir en colonización y/o infección del RN e incluso brotes infecciosos por Klebsiella pneumonia con $\beta$-lactamasa de espectro extendido (BLEE), Enterococcus spp. resistente a vancomicina y otros, lo que está directamente relacionado al uso no racional de $\mathrm{ATMs}^{17,18}$. Así, las infecciones con bacterias resistentes llevan a un aumento de la morbimortalidad y de los costos de la atención médica ${ }^{19}$.

La exposición del RN a ATMs después del nacimiento causa una desviación de los patrones de colonización normales con alteraciones en la diversidad y empobrecimiento de la microbiota intestinal, diferencias que pueden persistir hasta un año $^{20}$. La pérdida de diversidad ocasionada por los ATMs, especialmente en los primeros seis meses de vida -los más críticos para el desarrollo inmune- permite la proliferación de patógenos y secundariamente el subdesarrollo del sistema inmune adaptativo, ocurriendo cambios metabólicos permanentes con una pérdida de biodiversidad, que predispone al hospedero a un mayor riesgo de enfermedades infecciosas ${ }^{19}$. A mayor tiempo de exposición a ATMs, se disminuye en forma más acentuada la microbiota y la recuperación a niveles normales se enlentece y se entorpece. Además, el uso de antimicrobianos está asociado a displasia broncopulmonar, asma y obesidad ${ }^{21-23}$ y es conocido que algunos ocasionan toxicidad oto-nefrológica ${ }^{24}$.

Dado lo anterior, se ha creado la necesidad de tener una regulación y control de medicamentos en forma continua para mejorar la seguridad y calidad en la atención del paciente. Así se llega al concepto de antimicrobial stewardship, (administración adecuada de antimicrobianos) concepto ya instalado desde hace más de una década y cuyo objetivo fundamental es racionalizar el uso de antimicrobianos, es decir, hacer un trabajo dinámico, colaborativo y autocrítico de la forma de administrar medicamentos ${ }^{25,26}$. Los profesionales que integran el equipo de manejo racional de antimicrobianos dependen del tamaño del centro hospitalario, contando idealmente con un Neonatólogo, Infectólogo, Microbiólogo, Farmacéutico Clínico y personal de enfermería ${ }^{27-29}$. Desde el año 2011 se lleva un registro de uso de antimicrobianos en el Servicio de Recién Nacidos (SRN) del Complejo Asistencial Dr. Sótero del Río.

El objetivo de este estudio fue evaluar y describir el uso de los ATMs más empleados en un SRN de alta complejidad del sistema público en Chile. Adicionalmente, fue evaluar si en igual período se observaron cambios en la susceptibilidad de los microorganismos más frecuentes a los distintos ATMs. Este estudio pretende cuantificar también su uso con el fin de entregar información que sea una alerta ante su prescripción indiscriminada.

\section{Pacientes y Métodos}

Estudio de cohorte, prospectivo, observacional, unicéntrico, realizado en un hospital de atención terciaria de alta complejidad que, durante los años 2011 al 2014, tuvo aproximadamente 7.000 partos anuales. El SRN tiene 1.400 hospitalizaciones cada año para lo que se dispone de 54 camas, siendo 12 de cuidados intensivos.

\section{Criterios de inclusión}

Se contempló a la población total de $\mathrm{RN}$ hospitalizados en el período comprendido entre enero de 2011 y diciembre de 2014, pacientes de ambos géneros independientemente de su edad gestacional y diagnóstico de ingreso.

No se excluyó a ningún RN.

Para el registro de datos se utilizó una base en Microsoft Excel $^{\circledR}$ en la que se registró: peso de nacimiento, días de hospitalización, mortalidad, días de uso de los distintos antimicrobianos y otros fármacos de uso endovenoso, intramuscular u oral, excluyendo los tópicos como colirios, ungüentos, cremas, enjuagues, nebulizaciones o inhaladores. Adicionalmente, se registró el perfil de susceptibilidad antimicrobiana en cada cultivo realizado en el laboratorio de microbiología del hospital, para las 
bacterias más frecuentes: Staphylococcus coagulasa negativa (SCN) (Staphylococcus epidermidis y Staphylococcus capitis) y bacilos gramnegativos (BGNs). Se consideró la susceptibilidad antimicrobiana para cada cultivo, independientemente si se trataba del mismo paciente, con el fin de identificar también los cambios que presentaban durante su evolución clínica.

Se compararon años consecutivos; 2011-2012, 20122013 y 2013-2014 para evaluar si existieron variaciones de conducta a través de los años.

Para el análisis cuantitativo de consumo terapéutico de ATMs se utilizó la tasa global de ATMs (TUA) sumando el número de días que el $\mathrm{RN}$ estuvo expuesto a uno o más antibacterianos por cada 100 días de paciente hospitalizado. Para el cálculo de TUA no se incluyeron los ATMs de uso profiláctico, ${ }^{912}$. En forma concomitante, se creó otro registro de uso de ATMs sumando todos los días de ATMs utilizados durante cada año por 100 días de hospitalizados (STUA) como también por separado para los ATMs más frecuentemente administrados, a través de una tasa cuyo denominador son los días de uso de ATM, usualmente denominado días de terapia o DOT, y en el numerador los días de hospitalización calculados por 100 días. Se consideró día hospitalizado si el RN pernoctaba en el SRN y día de ATM si estaba medicado al momento de hacer el registro diario matinal. En el cálculo de uso individual se incluyó cefadroxilo porque es un ATM de frecuente uso en nuestro SRN como profilaxis de infección del tracto urinario (ITU). La metodología de cuantificación individual de uso de cada ATM fue elegida por la simpleza y factibilidad de hacerla, dado que existe amplia variación de peso en la población estudiada. La medida de cuantificación de ATM utilizada en adultos -dosis diaria definida o DDD- si bien existen reportes aislados en pediatría, no está validado para $\mathrm{RN}^{9,12}$. El análisis de uso de los ATMs más frecuentes por cada período mencionado se realizó para la población total del SRN y por subdivisión en rangos de peso de nacimiento al nacer: $<750 \mathrm{~g}, 750-1.000 \mathrm{~g}, 1.000-1.500 \mathrm{~g} \mathrm{y}>1.500 \mathrm{~g}$.

El equipo de trabajo estuvo formado por médicos tratantes, un infectólogo, el médico jefe de servicio y matronas que llevaron el registro de consumo de ATMs.

La decisión de iniciar una terapia antimicrobiana cuando existió sospecha de infección y cuando se consideró necesaria una profilaxis de ITU la adoptó el médico tratante o el médico residente.

El esquema utilizado está basado en la epidemiología local de nuestro SRN; para sepsis precoz, aquella instalada en menos de $72 \mathrm{~h}$ de vida, es ampicilina más gentamicina y para sepsis tardía, $\geq 72 \mathrm{~h}$ de vida, los ATMs usados son cloxacilina más amikacina y luego vancomicina, pipericilina/tazobactam, carbapenémicos y metronidazol. Otros, como cotrimoxazol y linezolid son antimicrobianos de uso excepcional y, por lo tanto, no se registró su administración. Por otro lado, como ATM profiláctico sólo se registró cefadroxilo dado que en nuestro SRN es de uso frecuente.

Este estudio fue presentado y aprobado por el Comité de Ética del Complejo Asistencial Dr. Sótero del Río. No se consideró la necesidad de consentimiento informado.

\section{Análisis estadístico}

La distribución de las variables se evaluó mediante el Test de Shapiro-Wilk. Las variables continuas se expresan en media \pm desviación estándar. Las variables categóricas se expresan en porcentaje. Además, los días de uso de ATM se expresan en una tasa por 100 días de hospitalización. La comparación de tasas obtenidas para los distintos períodos y la comparación de variables cualitativas se realizó por medio del test exacto de Fisher. Adicionalmente, para el análisis de las tendencias en el tiempo se realizó una regresión de Prais-Winsten para evaluación de tendencias lineales. En todas las comparaciones se consideraron diferencias significativas con un valor de $<0,05$. El análisis estadístico fue realizado con SPSS 14.6.

\section{Resultados}

Durante el período de registro se incorporaron 5.619 pacientes, existiendo 1.151 pacientes hospitalizados en el servicio el año 2011, 1.556 durante el año 2012, 1.426 el año 2013 y 1.486 el año 2014.

No se observaron diferencias en la distribución de los distintos rangos de peso durante los cuatro años registrados, perteneciendo cerca de $90 \%$ de los pacientes a la categoría de peso superior a $1.500 \mathrm{~g}$. Las características demográficas de los pacientes estudiados se muestran en la Tabla 1.

Durante los cuatro años de registro, 2.159 pacientes hospitalizados recibieron algún tipo de medicamento, representando $38,4 \%$ del total de pacientes. De los antimicrobianos administrados, 32,3\% correspondía a antibacterianos, $2,3 \%$ a antifúngicos y $0,9 \%$ a antivirales (Figura 1).

La cuantificación de consumo de ATMs, evaluado con la medición TUA desde el año 2011 al 2014, no evidenció un cambio significativo en tendencia de uso de ATM, oscilando entre 21,6 y 24,3\%. En la STUA se observó una disminución de 10,7\% entre 2011 y 2014 $(\mathrm{p}<0,05)$. Las variaciones en los distintos esquemas de ATM para sepsis tardía no fueron significativas en el análisis de los cuatro años (Figura 2). Por otro lado, la cantidad de uso de ATMs fue más alta con menor peso de nacimiento (Tabla 2).

Como ATM de primera línea destacó el uso de ampicilina que representó 19,1 a $23,9 \%$ del total de ATM 


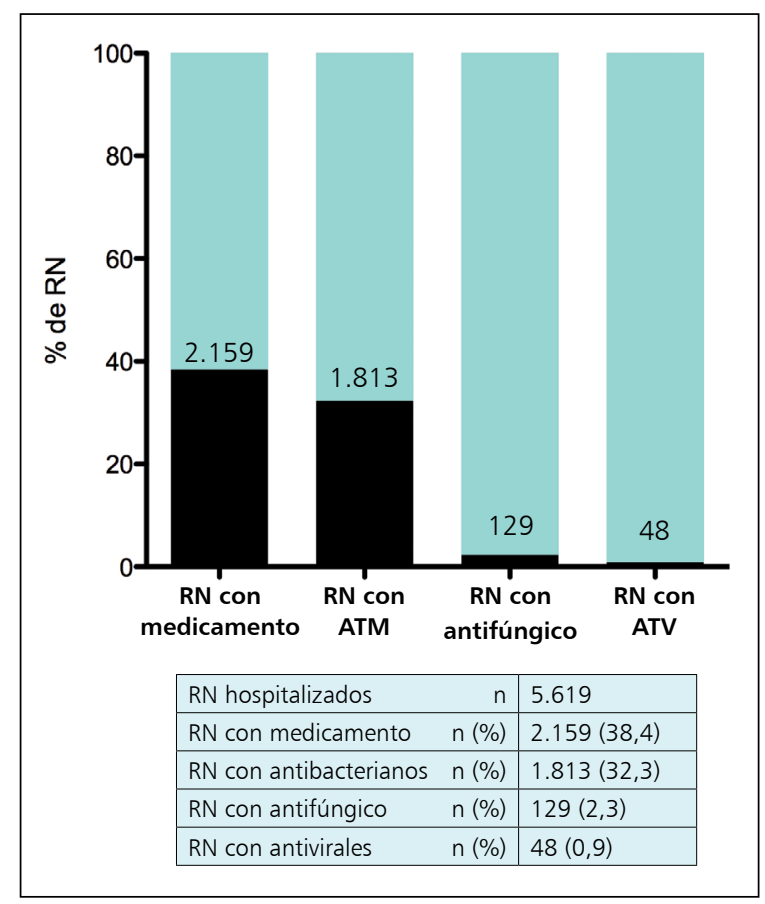

Figura 1. Distribución porcentual de uso de medicamentos y de antimicrobianos en el total de los recién nacidos hospitalizados entre el año 2011 y 2014. ATM: antimicrobiano, ATV: antiviral.

utilizado entre los años 2011 y 2014; más aún, sumándole a ésta el uso de gentamicina, en conjunto representaron casi $40 \%$ del total. En el período de registro, ampicilina presentó una tasa de uso entre 10,0 y 11,6 por 100 días de hospitalización (Figura 2). El uso de cefadroxilo fluctuó entre 5,6 y 7,6 por 100 días de hospitalización, representando $11,7 \%$ del total de ATMs usado.

La variación de uso ATMs durante los cuatro años observados fue acotada sólo a ciertos grupos de peso al nacer.

Para vancomicina, el uso en el grupo $<750 \mathrm{~g}$ al nacer la tasa de uso bajó en 2,7 y 2,6 días por 100 días de hospitalización, respectivamente $(\mathrm{p}<0,05)$ sin encontrarse diferencias en los otros grupos de peso.

En el análisis de uso de pipericilina/tazobactam, el grupo de peso al nacer $>1.500 \mathrm{~g}$ presentó un aumento significativo de 0,4 días por 100 días de hospitalización $(\mathrm{p}<0,05)$ a diferencia de todos los otros grupos de peso donde se observó una tendencia al aumento de uso, que no fue significativa.

Para metronidazol, la tendencia fue a aumentar su uso en 0,3 días por 100 días hospitalización en el grupo con peso menor de $750 \mathrm{~g}(\mathrm{p}<0,05)$, a diferencia del grupo de $\mathrm{RN}>1.500 \mathrm{~g}$ que por cada año descendió el uso en 0,2 días por 100 días hospitalización $(\mathrm{p}<0,05)$.
Tabla 1. Características generales de los recién nacidos hospitalizados entre 2011-2014. Complejo Asistencial Dr. Sótero del Río (n: 5.619)

\begin{tabular}{|c|c|c|c|}
\hline RN vivos & & $\mathrm{n}$ & 27.322 \\
\hline RN hospitalizados & & n (\%) & $5.619(20,6 \%)$ \\
\hline Género femenino & & $\mathrm{n}(\%)$ & $2.613(46,5 \%)$ \\
\hline Peso de nacimiento & gramos media & \pm DS & $2.829 \pm 874$ \\
\hline Edad gestacional & semana media & $\pm D S$ & $36,2 \pm 3,6$ \\
\hline Días hospitalizado global & mediana ( $r$ & (ango) & $4(0-371)$ \\
\hline RN & $<750 \mathrm{~g}$ & n (\%) & $93(1,7 \%)$ \\
\hline Días hospitalizado & $<750 \mathrm{~g}$ mediana $(r$ & (ango) & $75(7-97)$ \\
\hline RN & $750-999 \mathrm{~g}$ & n (\%) & $128(2,3 \%)$ \\
\hline Días hospitalizado & 750-999 g mediana ( $\mathrm{r}$ & (ango) & $59(27-82)$ \\
\hline $\mathrm{RN}$ & $1.000-1.499 \mathrm{~g}$ & $n(\%)$ & $304(5,41 \%)$ \\
\hline Días hospitalizado & $1.000-1.499 \mathrm{~g}$ mediana $(\mathrm{r}$ & (ango) & $41(26-55)$ \\
\hline RN & $>1.500 \mathrm{~g}$ & $n(\%)$ & $5.074(90,3 \%)$ \\
\hline Días hospitalizado & $>1.500 \mathrm{~g}$ mediana $(\mathrm{r}$ & (ango) & $3(2-8)$ \\
\hline Fallecen hospitalizados & & $n(\%)$ & $130(2,3 \%)$ \\
\hline Fallecimiento asociado a IAAS & & $\mathrm{n}(\%)$ & $40(0,7 \%)$ \\
\hline
\end{tabular}

RN, recién nacido, Edad gestacional en semanas (sem), Peso de nacimiento en gramos (g), IAAS, infección asociada a atención de salud.

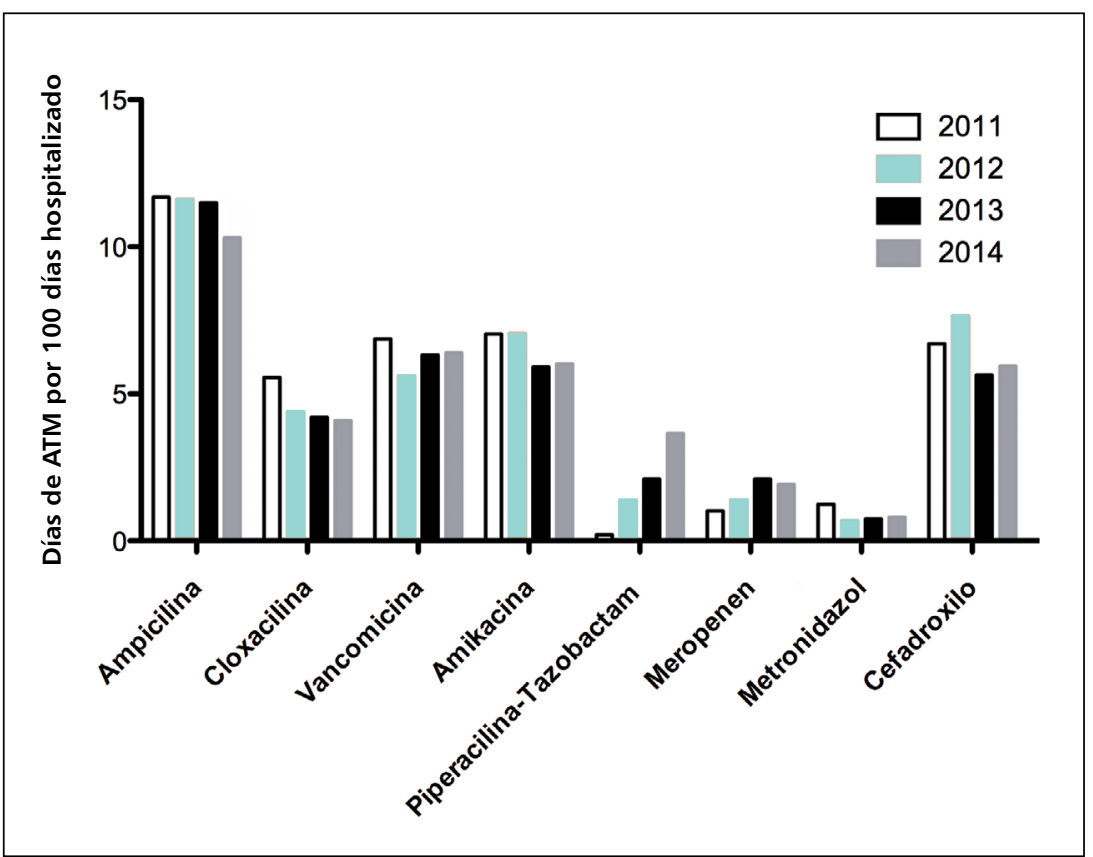

Figura 2. Exposición a los 8 antimicrobianos de uso más frecuente en todos los recién nacidos hospitalizados entre 2011 y 2014 medido como días de uso de antimicrobiano por 100 días de hospitalización. ATM: antimicrobiano. No se incluyó en el gráfico gentamicina por ser de uso concomitante a ampicilina como esquema de primera línea en sepsis precoz. 


\begin{tabular}{|c|c|c|c|c|}
\hline & Amikacina & Cloxacilina & Vancomicina & Meropenen \\
\hline$<$ de $750 \mathrm{~g}$ & $\begin{array}{c}9,73 \\
(9,02 \text { a } 10,49)\end{array}$ & $\begin{array}{c}6,51 \\
(5,92 \text { a } 7,15)\end{array}$ & $\begin{array}{c}19,70 \\
(18,73 \text { a } 20,71)\end{array}$ & $\begin{array}{c}6,36 \\
(5,78 \text { a } 7)\end{array}$ \\
\hline $750-999 \mathrm{~g}$ & $\begin{array}{c}12,11 \\
(11,42 \text { a } 12,83)\end{array}$ & $\begin{array}{c}5,70 \\
(5,22 \text { a } 6,22)\end{array}$ & $\begin{array}{c}14,60 \\
(13,87 \text { a } 15,4)\end{array}$ & $\begin{array}{c}3,38 \\
(3,01 \text { a } 3,79)\end{array}$ \\
\hline $1.000-1.499 \mathrm{~g}$ & $\begin{array}{c}7,74 \\
(7,3 \text { a } 8,21)\end{array}$ & $\begin{array}{c}5,34 \\
(4,97 \text { a } 5,74)\end{array}$ & $\begin{array}{c}5,63 \\
(5,25 \text { a } 6,04)\end{array}$ & $\begin{array}{c}1,00 \\
(0,85 \text { a } 1,19)\end{array}$ \\
\hline Sobre $1.500 \mathrm{~g}$ & $\begin{array}{c}5,16 \\
(4,94 \text { a } 5,39)\end{array}$ & $\begin{array}{c}4,16 \\
(3,96 \text { a } 4,37)\end{array}$ & $\begin{array}{c}2,55 \\
(2,39 \text { a } 2,71)\end{array}$ & $\begin{array}{c}0,58 \\
(0,51 \text { a } 0,66)\end{array}$ \\
\hline Global & $\begin{array}{c}7,01 \\
(6,82 \text { a } 7,21)\end{array}$ & $\begin{array}{c}4,82 \\
(4,66 \text { a } 4,99)\end{array}$ & $\begin{array}{c}6,36 \\
(6,18 \text { a } 6,55)\end{array}$ & $\begin{array}{c}1,58 \\
(1,49 \text { a } 1,68)\end{array}$ \\
\hline
\end{tabular}

Figura 3. Susceptibilidad comparativa entre Staphylococcus coagulasa negativa (SCN) años 2011 y 2014. Información de 200 cultivos en $172 \mathrm{RN}$.

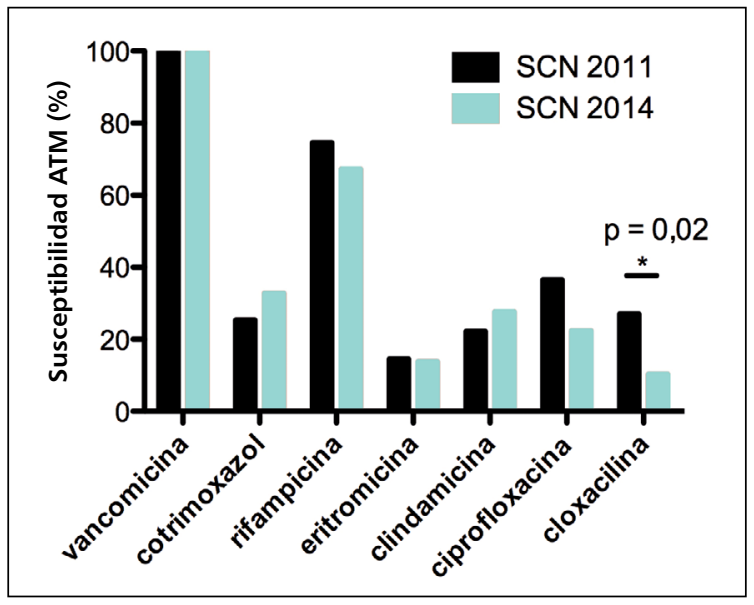

El análisis de tendencia no mostró diferencias en consumo de meropenem, amikacina y cloxacilina, en ninguno de los grupos observados ( $\mathrm{p}>0,05)$.

En relación a antimicóticos, el uso de fluconazol bajó desde 3,7 a 1,6 por 100 días de hospitalización y la tendencia por cada año fue a disminuir en 0,5 días por 100 días de hospitalización en el total de $\mathrm{RN}(\mathrm{p}<0,05)$. $\mathrm{Al}$ analizar por grupo de peso esta disminución fue de 0,7 día por 100 días hospitalización para el grupo de 750 a $1.000 \mathrm{~g}(\mathrm{p}<0,05)$. Para anfotericina, los días de uso fluctuaron entre 0,3 y 1,1 por 100 días de hospitalización ( $\mathrm{p}>0,05)$, pero sólo en el grupo de 1.000 a $1.499 \mathrm{~g} \mathrm{se}$ observó una disminución de uso de 0,1 día por 100 días de hospitalización $(\mathrm{p}<0,05)$.

El análisis de la susceptibilidad antimicrobiana para SCN no mostró cambios relevantes en los distintos años. El análisis de 200 cultivos en 172 RN que comparó el año 2011 con el 2014, mostró una disminución de la susceptibilidad a cloxacilina desde 27 a 10,3\% (p < de 0,05) (Figura 3).
Al evaluar 272 cultivos de 175 RN entre el año 2011 y el año 2014 se observó que en los BGN existió una disminución significativa en la sensibilidad a cefotaxima, ceftazidima, gentamicina y ciprofloxacina $\mathrm{p}<0,05$; $\sin$ embargo, hubo un aumento significativo en la susceptibilidad in vitro para amikacina y pipericilina/tazobactam $(\mathrm{p}<$ de 0,05$)$ (Figura 4a). Si se analiza a los BGN excluyendo a Klebsiella pneumoniae, se observó aumento en la actividad de ceftazidima, gentamicina y amikacina al comparar los años 2011 y 2014 (p < 0,05) (Figura 4b). Analizando separadamente sólo Klebsiella pneumoniae en los 151 cultivos tomados a 87 RN entre 2011 y 2014 , hubo una disminución significativa de la susceptibilidad a cefotaxima, ceftazidima y gentamicina $(\mathrm{p}<0,05)$. También se observó un aumento en la susceptibilidad para amikacina y pipericilina/tazobactam entre 2011 y $2014(\mathrm{p}<0,05)$ (Figura 4c).

Al analizar los cultivos, se observó que, en 2011, 6,7\% de los BGN aislados en $89 \mathrm{RN}$ fueron productores de BLEE, en comparación a $49 \%$ de los cultivos realizados a $86 \mathrm{RN}$ durante el año 2014. De igual forma, los pacientes con aislamiento de BGN presentaron un aumento de cultivos positivos a Klebsiella pneumoniae de $38 \%$ en el 2011 y de $50 \%$ durante el 2014 siendo productores de BLEE 17,6 y $75,5 \%$, respectivamente.

En relación a la mortalidad intrahospitalaria asociada a infecciones asociadas a la atención en salud (IAAS), no se observó cambios entre los años 2011 y 2013. El año 2014, aumentó la tasa de enterocolitis necrosante en la unidad, de manera concomitante a un brote epidémico causado por Klebsiella pneumoniae, el que se inició en febrero de 2014 y finalizó ese mismo año con tres semanas epidemiológicas continuas sin casos nuevos. El brote afectó a 32 pacientes con 39 episodios infecciosos, de los cuales $74 \%$ fueron de foco urinario. En este brote falleció un RN lo que representó una tasa de letalidad de $2,6 \%$. 


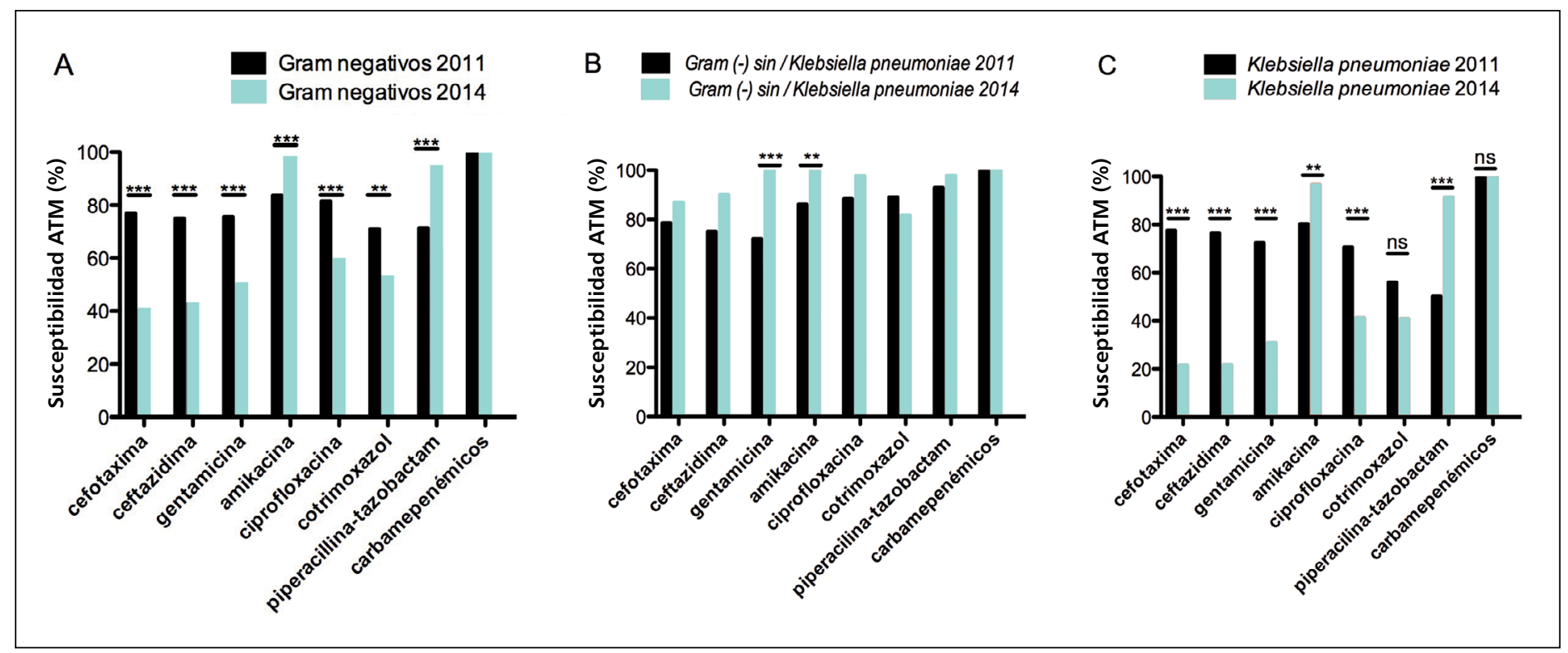

Figura 4. Susceptibilidad comparativa de bacilos gramnegativos entre año 2011 y 2014 . Información de 272 cultivos de 175 RN. A) Susceptibilidad comparativa de todos los bacilos gramnegativos. B) Susceptibilidad comparativa entre bacilos gramnegativos exceptuando Klebsiella pneumoniae. C) Susceptibilidad comparativa entre Klebsiella pneumoniae. ${ }^{* *} p<0,05$, ns: no significativo.

\section{Discusión}

Este estudio de cuatro años muestra que, aproximadamente $40 \%$ de los RN hospitalizados reciben medicamentos y que un porcentaje mayoritario de estos corresponden a antibacterianos, lo que es coincidente con lo descrito en otros centros de atención neonatal ${ }^{1,9}$.

No se observó cambios significativos en el consumo global de ATM evaluado como TUA en el período observado, como tampoco, según se observa en la Figura 2, hubo cambios importantes en el consumo de los ATM más frecuente utilizados en nuestro SRN. Si se analiza como sumatoria de uso total por año o STUA, sí existió disminución de 10,7\% entre 2011 y 2014 lo que probablemente se explica por un ajuste y suspensión más precoz del esquema ATM inicial según microbiología, llegando en forma más precoz a la monoterapia.

La combinación ampicilina + gentamicina fue el esquema ATM más usado por ser el tratamiento empírico de primera línea para sepsis precoz, dado que al igual que en otros países como E.U.A., lo consideramos como el tratamiento empírico estándar para cubrir los microorganismos más comunes de transmisión vertical como son Streptococcus agalactiae y Escherichia coli ${ }^{30}$.

Es de notar que en la población general de $\mathrm{RN}$ no se observó variación en el análisis de tendencia de uso de los ATMs prescrito para sepsis precoz y sepsis tardía, sólo encontrándose diferencias para el análisis si se consideran subgrupos de peso. Uno de los cambios más llamativos en este sentido, fue el aumento de la utilización de pipericilina/tazobactam desde 2011 a 2014 en el $\mathrm{RN}>1.500$ g al nacer; esto es explicable dado que se incorporó este ATM al arsenal terapéutico desde esa fecha (Figura 2). También se descontinuó paulatinamente la utilización de imipenem, manteniéndose sólo meropenem dentro de los carbapenémicos. Esta es una de las razones por la que probablemente existe tendencia a aumentar su uso en un grupo de peso. Para el caso puntual de meropenem, estimamos que de aumentar el tamaño muestral y el tiempo de seguimiento la tendencia sería a aumentar el uso en el grupo de 1.000 a $1.499 \mathrm{~g}$.

Metronidazol mostró un aumento de su utilización en el grupo con peso menor de $750 \mathrm{~g}$. Sin embargo, debido a la consideración del reducido tamaño de la muestra, se dificulta la interpretación de los datos.

El uso de vancomicina disminuyó sólo en el grupo de menos de $750 \mathrm{~g}$ al nacer, probablemente como consecuencia de mayor vigilancia y esfuerzo focalizado en este grupo de pacientes. No se observó lo mismo en otros subgrupos y el consumo global no presentó variaciones en el período observado, lo que revela que vancomicina sigue utilizándose en nuestro medio pese al comprobado mayor riesgo de adquisición de hongos y de la emergencia de Enterococcus resistente a vancomicina. A pesar de los esfuerzos, sigue siendo una necesidad hacer más énfasis en restringir su uso, dejándola para pacientes muy acotados como: prematuros extremos, graves y para Staphylococcus aureus resistente a cloxacilina. Existe 
amplia literatura médica que apoya el uso de cloxacilina como antimicrobiano de primera elección en sepsis tardía, incluso en SCN resistentes a cloxacilina in vitro, no siendo necesario escalar a vancomicina si se obtiene buena respuesta clínica, si se procede al retiro de catéteres y si parte de la terapia incluyó aminoglucósidos que, teóricamente actuarían como sinérgicos a cloxacilina para este microorganismo ${ }^{17,31}$

También consideramos relevante la observación del alto uso de cefadroxilo, (11,7\% del total de uso de ATMs), dado la descripción cada vez más frecuente del cambio en la resistencia de los uropatógenos en los pacientes que usan uroprofilaxis. Es un tema a reevaluar y hacer guías de uso de profilaxis con ATMs en pacientes que han presentado infecciones del tracto urinario o con alteraciones ecográficas fetales de la vía urinaria ${ }^{32}$.

Al evaluar la susceptibilidad de SCN, no se observan diferencias, excepto la disminución de susceptibilidad a cloxacilina durante el año 2014. El continuar usando vancomicina puede haber seleccionado cepas de SCN con presencia del gen mecA que le proporciona el potencial de resistencia a meticilina (cloxacilina) ${ }^{17}$.

Los cambios en susceptibilidad a ATMs en BGNs, si se excluye a Klebsiella pneumoniae, tampoco son relevantes. Sin considerar Klebsiella pneumoniae. Es posible observar en los otros BGNs (Figura 4-B) un aumento en la susceptibilidad a aminoglucósidos al comparar los años 2011 y 2014. Esto lo podemos explicar posiblemente porque el incremento de Klebsiella pneumoniae que progresivamente se constituyó en el BGN predominante en el centro, instó a introducir piperacilina/tazobactam. Este ATM presenta una alta actividad in vitro sobre todos los BGNs (aproximadamente $80 \%$ en nuestra institución); su uso posiblemente eliminó poblaciones que poseían resistencia a otros ATMs, considerando la posibilidad que las bacterias gramnegativas pueden ser de poblaciones mixtas con susceptibilidades diferentes.

Si se analiza separadamente sólo Klebsiella pneumoniae, se observó cambio en el patrón de susceptibilidad a cefotaxima, ceftazidima, ciprofloxacina y gentamicina con un aumento porcentual de resistencia a esos ATMs. Además, dentro del período observado, hubo brote de Klebsiella pneumoniae con un incremento significativo de bacterias productoras de BLEE, lo que, podemos especular, explica la mayoría de los cambios de susceptibilidad de los BGNs en el período observado. Es conocido que el uso de ATM permite la generación de líneas evolutivas de enzimas hacia variantes de mayor espectro con mutaciones adicionales en el sitio activo que permiten la aparición de resistencia a cefalosporinas de segunda y tercera generación $^{33}$. Como describe Rettedal, el uso de ATM y la edad gestacional al nacer antes de las 37 semanas de edad gestacional, son factores independientes para la colonización por Klebsiella pneumoniae productora de $\mathrm{BLEE}^{18}$.
Es claro que este estudio tiene limitaciones, como es el tener un registro registro manual de uso de medicamentos, que depende del personal tratante $\mathrm{y}$, en este sentido, es altamente probable que exista un sub-registro del uso de medicamentos. Adicionalmente, es un reporte unicéntrico, lo que entregando una buena mirada institucional, nuestros resultados no son extrapolables a otros lugares, a pesar de que es conocida la dispersión en los días de uso de ATM en múltiples centros. Existen trabajos recientes, como el de Schulman y cols. ${ }^{9}$, donde utilizando una medición de días de uso de antimicrobiano por 100 días de hospitalización, constataron una dispersión muy alta en las tasas de uso de ATM en los diversos centros de atención neonatal. Este fenómeno observado fue independiente del grado de complejidad de los pacientes, del tipo de patologías médicas o quirúrgicas que presentaban y si se trataba de infección comprobada o no. Esta conducta ya ha sido visualizada previamente, observándose que las tasas de uso de antimicrobianos tienen más relación con las prácticas locales que con las distintas co-morbilidades de los pacientes y que situaciones como brotes de infecciones bacterianas aumentan la prescripción de ATM de tercera línea en los seis meses posteriores ${ }^{9,34,35}$.

Otro aspecto relevante y que representa un problema para la comparación entre centros similares, es la falta de estandarización de la herramienta de medición del uso de ATMs. La alternativa utilizada en adultos es la “dosis diaria definida" (DDD), que está estandarizada para adultos de $70 \mathrm{~kg}$, herramienta no validada para la población pediátrica y neonatal. En el neonato existen diversas formas de medición de uso de ATM como "dosis diarias definidas por peso", útil sobre todo dada la gran dispersión de pesos en RN. Otra medida que parece ser efectiva y más simple, es generar una tasa de días de uso de ATM en hospitalizados, porcentaje de uso, como lo hace el grupo de Schulman y cols., en California ${ }^{9,36}$, o como lo realizan Ting y cols., de la red canadiense, al calcular el número de días de exposición a uno o más ATMs dividido por el número total de días hospitalizado. Este último autor excluye los ATMs usados para profilaxis como cefadroxilo, cotrimoxazol y otros ${ }^{12}$. Más allá de las distintas formas de registro, sigue siendo muy importante evaluar en forma permanente la tasa de uso de ATMs, debiendo siempre ir ligado a una retroalimentación local. Es recomendable realizar comparaciones entre los distintos centros e ir evaluando todas las intervenciones que se efectúan como lo muestran en su artículo Cantey y cols., quienes logran disminuir en forma segura el uso de $\mathrm{ATMs}^{36}$. Por lo anterior, sería recomendable un registro de uso estandarizado de todos los ATMs, tanto programas locales como nacionales, para llevar un adecuado seguimiento y control de su uso, lo que favorecería la disminución de la resistencia a ATMs y haría posible realizar intervenciones en caso de uso inadecuado. 
Este estudio, de carácter observacional, pretende objetivar el consumo de ATM de un SRN. Tiene la fortaleza de mostrar que se puede llevar un registro simple con una medida de consumo de ATMs usando tasas de uso global como es TUA y además, muestra que puede hacerse la vigilancia de los ATM más utilizados y los que se consideren afectan más el ambiente de los microorganismos hospitalarios, como es vancomicina ${ }^{18,33}$. Nos permite analizar nuestra realidad de consumo de ATMs y la susceptibilidad de los microorganismos y nos motiva a organizar, no siendo sólo un ente observacional, un programa de uso racional de ATM para disminuir en forma segura las tasas de uso innecesario de ATM. Experiencias aisladas, como la de Romaniszyn y cols., hacen vigilancia activa de las infecciones por $S$. aureus, su susceptibilidad in vitro y el consumo de $\mathrm{ATM}^{37}$.

En conclusión, se puede llevar un registro del uso de ATMs y de susceptibilidad a antimicrobianos. Nuestra recomendación es que se analice el uso global de ATMs, para cada antimicrobiano por separado, y por rangos de peso, incluyendo las cefalosporinas utilizadas para profilaxis de ITU. Este tipo de información puede ser de gran utilidad para el mejor manejo de los pacientes, especialmente si se hace como una retro-alimentación permanente que mantenga y refuerce conductas en las decisiones médicas que van a favorecer al paciente y, en lo posible, disminuir la alteración del ecosistema bacteriano, fortaleciendo las "conductas inteligentes" en el uso de ATM. Sin embargo, queda claro que tanto el registros como los cambios de conducta y el control en el uso de ATMs deben ser considerados una tarea multidisciplinaria que reúna a todos los profesionales de la salud que se desenvuelven en unidades de intensivos neonatales. Es necesario enfatizar que para poder realizar el "stewardship de antimicrobianos" se necesita profesionales que trabajen en equipo, con información actualizada y con tiempo asignado para esta tarea.

Agradecimientos. Especiales a Sra. Eliana Correa y Sofía Gallardo por su gentileza y colaboración en la recopilación de información. A Pablo Sánchez, estudiante de ingeniería de Universidad del Desarrollo por su ayuda logística y a Dagoberto Ojeda, Médico Anestesista de Clínica Dávila.

\section{Resumen}

Antecedentes: Los antimicrobianos (ATM) son uno de los medicamentos más utilizados en recién nacidos (RN) hospitalizados. El uso indiscriminado de ATM trae consecuencias negativas como son el predominio de bacterias resistentes a los ATM usualmente utilizados y asociaciones individuales a morbilidad relevante como son la displasia broncopulmonar, enterocolitis necrosante, sepsis tardía y/o muerte. Objetivo: Registrar y evaluar las tendencias del uso de ATM a lo largo del tiempo en RN hospitalizados en el Servicio de Neonatología (SRN) del Complejo Asistencial Dr. Sótero del Río, con el fin de objetivar los cambios en la práctica habitual de la indicación de ATM. Un objetivo secundario fue evaluar el impacto de estas conductas sobre la resistencia antimicrobiana. Métodos: Estudio de cohorte, prospectivo, observacional, unicéntrico, en todos los pacientes hospitalizados entre enero de 2011 y diciembre de 2014. Se registró el peso al nacer, días de hospitalización, indicación y días de uso de ATM para cada paciente. El uso de ATM fue cuantificado por medio de distintas tasas: días de indicación de un o más ATM para el consumo global (TUA), sumatoria total de días de uso (STUA) como para los ATM más frecuentemente utilizados. Cada tasa calculada por 100 días hospitalizados. Además, se registró la susceptibilidad antimicrobiana de las bacterias más frecuentemente aisladas en nuestro servicio: Staphylococcus coagulasa negativa (SCN) y bacilos gramnegativos (BGNs). Resultados: El 34,7\% de los pacientes hospitalizados recibió algún tipo de antimicrobiano, correspondiendo 32,3\% a antibacterianos. El ATM más utilizado fue ampicilina (20,2\% del total) y luego cefadroxilo (11,6\%). El TUA no cambió entre 2011 y 2014. La STUA disminuyó en $10,7 \%$ entre 2011 y 2014 ( $p<0,05)$. En el análisis por rangos de peso, en el grupo $<750 \mathrm{~g}$ disminuyó la tendencia de uso de vancomicina (descenso de uso en $9,9 \%$ ) y un aumento de $18,8 \%$ para metronidazol. Por otra parte, hubo un aumento en el uso del régimen de piperacilina/tazobactam en el grupo $>1.500 \mathrm{~g}$. Al evaluar la susceptibilidad antimicrobiana, hubo una disminución de la susceptibilidad a cloxacilina en SCN entre 2011 y 2014 desde 27 a 10,3\%, respectivamente. Para BGN hubo una disminución desde 76,9 a 40,5\% en la susceptibilidad a cefalosporinas de tercera generación, principalmente debido a Klebsiella pneumoniae que pasó a ser el BGN predominante, con un aumento de 6,7 a $50 \%$ en los años 2011 y 2014, respectivamente. Para Klebsiella pneumoniae la susceptibilidad a cefalosporinas de tercera generación descendió desde 77 a 22\%. Por último, amikacina mostró una actividad sobre $85 \%$ en todos los BGNs entre 2011 y 2014. Conclusiones: Es recomendable planificar y mantener un registro continuo del consumo de ATM tanto como terapia y profilaxis, idealmente llevar el TUA, el STUA y siendo categorizado por tipo de ATM y rango de peso de los RN. En forma concomitante, es de considerable importancia analizar y evaluar la susceptibilidad de microorganismos. Es esencial que un equipo interdisciplinario prepare este registro, y que continuamente proporcione retroalimentación a los profesionales que mantienen el funcionamiento de las unidades de cuidados neonatales. 


\section{Referencias bibliográficas}

1.- Clark R H, Bloom B T, Spitzer A R, Gerstmann D R. Reported medication use in the neonatal intensive care unit: date from a large national data set. Pediatrics 2006; 117: 1979-87.

2.- Centers for Disease Control and Prevention. Antibiotic resistance threats in the United States, 2013 report. Available at: https:/www. cdc.gov/drugresistance/threat-report-2013/pdf/ ar-threats-2013-508.pdf\#page=11. (Accedido febrero 13, 2015)

3.- Fisher J E. Physicians' ability to diagnose sepsis in newborns and critically ill children. Pediatr Crit Care Med 2005; 6 (3): S120-5.

4.- Bekhof J, Reitsma J B, Kok J H, Van Straaten_I H. Clinical signs to identify late-onset sepsis in preterm infants. Eur J Pediatr 2013; 172: 501-8.

5.- Bennett N J, Tabarani C M, Bartholoma N M, Wang D, Huang D, Riddell S W, et al. Unrecognized viral respiratory tract infections in premature infants during their birth hospitalization: A prospective surveillance study in two neonatal intensive care units. J Pediatr. 2012 Nov; 161 (5): 814-8.

6.- Kidszun A, Hansmann A, Winter J, Grondahl B, Knuf M, Weise K, et al. Detection of respiratory viral infections in neonates treated for suspicion of nosocomial bacterial sepsis: A feasibility study. Pediatr Infect Dis J. 2014; 33: 102-4.

7.- $\quad$ Ronchi A, Michelow I C, Chapin K C, Bliss J M, Pugni L, Mosca F, et al. Viral respiratory tract infections in the neonatal intensive care unit: The VIRIoN-I study. J Pediatr. 2014; 165 : 690-6.

8.- Stoll Barbara J, Shane Andi L. Infections of the Neonatal Infant. Robert M. Kliegman Bonita F. Stanton, Joseph W. St Geme and Nina F. Schor. Nelson Textbook of Pediatrics, Twentieth Edition Elsiever, Phyladelphia. 2016; p 909-25.

9.- Schulman J, Dimand R, Lee H, Duenas G, Bennett M, Gould B. Neonatal Intensive Care Unit antibiotic use. Pediatrics 2015; 135: 82633.

10.- Arboleya S, Sánchez B, Milani C, Duranti $\mathrm{S}$, Solís G, Fernández N, et al. Intestinal microbiota development in preterm neonates and effect of perinatal antibiotics. J Pediatr 2015 Mar; 166 (3): 538-44.

11.- Greenwood C L, Morrow A, Lagomarcino A, Altaye M, Taft D H, Yu Z, et al. Early empiric antibiotic use in preterm infants is associated with lower bacterial diversity and higher relative abundance of Enterobacter. J Pediatr 2014: 165: 23-9.

12.- Ting J, Synnes A, Roberts A, Deshpandey A, Dow K, Yoon E, et al. Association between antibiotic use and neonatal mortality and morbidities in very low birth weight infants without culture proven sepsis or necrotizing enterocolitis. JAMA Pediatr 2016; 170 (12): 1181-7.

13.- Cotten C M, Taylor S, Stoll B, Goldberg R
N, Hansen N I, Sánchez P J, et al. Prolonged duration of initial antibiotic treatment is associated with increased rates of necrotizing enterocolitis and death for extremely low birth weight infants. Pediatrics 2009; 123: 58-66.

14.- Kuppala V S, Meinzen-Derr J, Morrow A L, Schibler K R. Prolonged initial empirical antibiotic treatment is associated with adverse outcomes in premature infants. J Pediatr 2011; 159: 720-5.

15.- Cotten C M, McDonald S, Stoll B, Goberg R, Poole K, Bejamin D K Jr. The association of third-generation cephalosporin use and invasive candidiasis in extremely low birth-weight infants. Pediatrics 2006; 118: 717-22.

16.- Lee J H, Hornik C P, Benjamin D K Jr, Herring A H, Clark R H, Cohen-Wolkowiez M, et al. Risk factors for invasive candidiasis in infants $>1500 \mathrm{~g}$ birth weight. Pediatr Infect Dis J 2013; 32: 222-6.

17.- Sandoval A 1, Cofré F, Delpiano L, Izquierdo G, Labraña, Reyes A. Reposicionando la cloxacilina como antibioticoterapia empírica inicial de la sepsis tardía neonatal Rev Chilena Infectol 2015; 32 (2): 182-9.

18.- Rettedal S, Hoyland Löhr I, Natås O, Sundsfjord A, Øymar K. Risk factors for acquisition of CTX-M-15 extended-espectrum beta-lactamase-producing Klebsiella pneumoniae during an outbreak in a neonatal intensive care unit in Norway. Scand J Infect Dis. 2013 Jan; 45 (1): 54-8.

19.- Vangay P, Ward T, Gerber J S, Knights D. Antibiotics, pediatric dysbiosis, and disease. Cell Host Microbe 2015 May 13; 17 (5): 55364.

20.- Simioni J, Huttom E K, Gunn E, Holloway A C, Stearns J C, McDonald H, et al. A comparison of intestinal microbiota in a population of low-risk infants exposed and not exposed to intrapartum antibiotics: The Baby \& Microbiota of the Intestine cohort study protocol. BMJ Pediatr 2016 Nov 10; 16 (1): 183.

21.- Novitsky A, Tuttle D, Locke R G, Saiman L, Mackley A, Paul D A. Prolonged early antibiotic use and bronchopulmonary dysplasia in very low birth weight infants. Am J Perinatol 2015; 32: 43-8

22.- Lapin B, Piorkowski J, Ownby D, WagnerCassanova C, Freels S, Chavez N, et al. The relationship of early-life antibiotic use with asthma in at-risk children. J Allergy Clin Immunol 2014; 134: 728-9.

23.- Saari A, Virta L J, Sankilampi U, Dunkel L, Saxen H. Antibiotic exposure in infancy and risk of being overweight in the first 24 months of life. Pediatrics 2015; 135: 617-26.

24.- Zappitelli M, Selewski D, Askenazi D. Nephrotoxic medication exposure and acute kidney injury in neonates. Neoreviews 2012; 13 (7): 420-7.

25.- Owens Jr R, Fraser G L, Stogsdill P. Antimicrobial stewardship programs as a means to optimize antimicrobial use. Insights from the Society of Infectious Diseases Pharmacists. Pharmacotherapy 2004 Jul; 24 (7): 896-908.

26.- Dellit T H, Owens R C, McGowan J E, Gerding D N, Weinstein R A, Burke J P, et al. Infectious Diseases Society of America and the Society for Healthcare Epidemiology of America guidelines for developing an institutional program to enhance antimicrobial stewardship. Clin Infect Dis 2007; 44: 159-77.

27.- Patel S J, Saiman L. Principles and strategies of antimicrobial stewardship in Neonatal Intensive Care Unit. Semin Perinatol 2012; 36 : 431-6.

28.- Patel S J, Saiman L. Antibiotic resistance in neonatal intensive care unit pathogens: mechanisms, clinical impact, and prevention including antibiotic stewardship. Clin Perinatol 2010; 37 (3): 547-63.

29.- Cantey J B, Patel S J. Antimicrobial stewardship in the NICU. Infect Dis Clin N Am 2014; 28: 247-61.

30.- Ramasethu J A, Kawakita T. Antibiotic stewardship in perinatal and neonatal care Semin Fetal Neonatal Med. 2017 Oct; 22 (5): 278-83.

31.- Chiu CH, Michelow I C, Cronin J, Ringer S A, Ferris T G, Puopolo K M. Effectiveness of guideline to reduce vancomycin use in neonatal intensive care. Pediatr Infect Dis J. 2011; 30 (4): 273-8.

32.- Bitsori M, Makari S, Galanakis E. Longterm resistance trends of uropathogens and association with antimicrobial prophylaxis. Pediatri Nephrol; 29 (6): 1053-8.

33.- Fica A. Resistencia antibiótica en bacilos gram negativos, cocáceas gram positivas y anaerobios. Implicaciones terapéuticas. Rev Med Clin Condes 2014; 25 (3) 432-44.

34.- Grohskopf L A, Huskins W C, Sinkowitz Cochrans R L, Levine G L, Goldmann D A, Jarvis W R. Pediatrics Prevention Network Use of antimicrobial agents in United States neonatal and pediatrics intensive care patients. Pediatr Infect Dis J 2005; 24 (9): 766-73.

35.- Hartel C, Hartz A, Bahr L, Gille C, Gortner L, Simon A et al. Media stories on NICU outbreaks lead to an increased prescription rate of third-line antibiotics in the community of Neonatal Care. Infect Control Hosp Epidemiol 2016; 37: 924-30.

36.- Cantey J B, Wosniak P S, Pruszynski J E, Sánchez P J. Reducing unnecessary antibiotic use in the neonatal intensive care unit (SCOUT): a prospective interrupted time-series study. Lancet Infect Dis 2016; 16 (10): 117884.

37.- Romaniszyn D, Rozanska A, Wojkowska-Mach J, Chmielarczyk A, Pobiega M, Adamski P, et al. Epidemiology, antibiotic consumption and molecular characterisation of Staphylococcus aureus - data from the Polish Neonatology Surveillance Network, 2009-2012 BMC Infect Dis. 2015 Apr 1; 15:169. doi: 10.1186/s12879015-0890-3. 